\title{
Construção de um Questionário Transcultural de Motivos de Abandono do Ensino Superior
}

\author{
Leandro S. Almeida, Joana R. Casanova \\ Universidade do Minho, Campus Gualtar, Portugal \\ Ana B. Bernardo, António Cervero \\ Universidade de Oviedo, Oviedo, Espanha \\ Acácia A. Angeli dos Santos ${ }^{1}$, Rodolfo A. M. Ambiel \\ Universidade São Francisco, Campinas-SP, Brasil
}

\section{RESUMO}

O abandono no Ensino Superior é um tema relevante pela sua incidência e consequências negativas no estudante, família, instituição e sociedade em geral. Procurando conhecer os motivos da intenção de abandono, investigadores do Brasil, Espanha e Portugal convergiram na construção de um questionário de avaliação transcultural. Atendendo à multidimensionalidade das causas de abandono, o questionário abarca seis dimensões: Social, Acadêmica, Professores, Saúde e Bem-estar, Institucional e Financeira. A análise fatorial, com amostras autônomas de estudantes do $1^{\circ}$ ano do Brasil, Espanha e Portugal, mostrou uma distribuição adequada dos itens nas seis dimensões, com alguma discrepância na dimensão Professores na amostra do Brasil. Os índices de consistência interna atingiram níveis adequados nas seis dimensões (valores entre 0,74 e 0,91 ). Futuros desenvolvimentos devem testar a dimensionalidade do questionário e a sua invariância nos três países, incluindo já variáveis psicológicas e académicas para análise da validade de critério.

Palavras-chave: ensino superior; motivos de abandono; estudantes do $1^{\circ}$ ano

\section{ABSTRACT - Development of a Transcultural Questionnaire of Motives for Higher Education Dropout}

The dropout in higher education assumes relevant percentages, with negative consequences for the students, family, institution and society in general. In order to know the reasons for the dropout intention, researchers from Brazil, Spain and Portugal converged on the construction of a transcultural questionnaire. Face the multidimensional nature of dropout causes, the questionnaire assesses six dimensions: Social, Academic, Faculty, Health and Wellbeing, Institutional, and Financial. The factorial analysis, with autonomous samples of first-year students from Brazil, Spain and Portugal, showed an adequate distribution of the items by the six dimensions, with some difficulties in Faculty dimension in Brazilian sample. The internal consistency of items reached adequate levels in those six dimensions (values range from .74 to .91). Future developments should test the dimensionality of the questionnaire and its invariance in all three countries, including other psychological and academic variables for criterion validity analysis.

Keywords: higher education; dropout reasons; first-year students.

RESUMEN - Construcción de un Cuestionario Transcultural de Motivos de Abandono de la Enseñanza Superior El abandono en la enseñanza superior es un tema relevante por su incidencia y por sus consecuencias negativas a nivel personal, familiar, institucional y social. En búsqueda de conocer las razones de intención de abandono, investigadores de Brasil, España y Portugal participaron en la construcción de un cuestionario de evaluación transcultural. Dicho instrumento, de naturaleza multidimensional, se organiza en seis dimensiones: Social, Académica, Profesorado, Salud y Bienestar, Institucional y Financiera. El análisis factorial, con muestras independientes de los estudiantes del primer año de Brasil, España y Portugal, mostró una distribución adecuada de los ítems en las seis dimensiones, con alguna discrepancia en la dimensión Profesorado en la muestra brasileña. Los índices de consistencia interna alcanzaron niveles adecuados (valores entre .74 y .91), si bien que se debe realizar nuevos estudios para probar la dimensionalidad del cuestionario y su invariancia en los tres países, incluyendo las variables psicológicas y académicas para analizar la validez de criterio.

Palabras clave: educación superior; motivos de abandono; estudiantes de $1^{\mathrm{o}}$ año.

A necessidade nas sociedades modernas de quadros superiores para responderem às solicitações do mercado de trabalho e à complexidade da vida social é pouco consentânea com as taxas anuais de estudantes que abandonam o ensino superior (ES). Por outro lado, não sendo um nível de ensino de frequência compulsiva, o abandono do ES traduz expetativas iniciais dos estudantes e das suas famílias não concretizadas, com os 
sentimentos de perda ou frustração inerentes. Esses sentimentos evidenciam-se, aliás, quando se reconhece que a formação acadêmica superior tende a ser um fator de melhor emprego e de proteção do desemprego na vida adulta (Eurostat, 2018). Finalmente, o abandono do ES traduz custos para a sociedade e para as próprias instituições de ensino superior (IES), neste último caso traduzidos em menor financiamento quando está associado ao número de estudantes e de diplomados. Todos esses aspetos destacam a relevância do tema abandono do ES e a necessidade de se conhecerem as causas para a definição de medidas preventivas mais adequadas (European Commission, 2015).

Dados relativos ao abandono nas IES públicas em Portugal mencionam taxas preocupantes. No quadro de um projeto de investigação tomando os estudantes que ingressaram pela primeira vez no ES em quatro universidades portuguesas (Porto, Minho, Lisboa, Minho, Évora) no ano letivo de 2009/2010, e acompanhados até ao final de $2013 / 14$, verificava-se que $32,4 \%$ dos inscritos tinham abandonado a sua instituição de ensino (possível que alguns tenham mudado de instituição), situando-se já em $18 \%$ a taxa de abandono verificada no final de 2009/2010, ou seja, no final do $1 .^{\circ}$ ano dos cursos (Almeida, Vasconcelos, \& Mendes 2018). Dados similares são fornecidos pela Direção-Geral de Estatísticas da Educação e Ciência (Engrácia \& Baptista, 2018) tomando os estudantes que, no ano letivo 2011/12, inscreveram-se no $1^{\circ}$ ano, pela primeira vez, em cursos de licenciatura de três anos. Esses estudantes foram acompanhados durante quatro anos (até 2014/15), registando-se que, passados quatro anos, $26 \%$ no ensino universitário público e $30 \%$ no ensino politécnico público não se encontravam no ensino superior (e não haviam concluído os seus cursos). De acrescentar que essas taxas de abandono diferem de acordo com as áreas científicas dos cursos, sendo maiores nos cursos de Educação e Humanidades, aliás, também maiores junto dos estudantes do sexo masculino e daqueles que ingressam no ES com piores classificações no ensino secundário.

No caso da Espanha, os dados são igualmente preocupantes e semelhantes aos de Portugal, com a taxa de abandono superior a $20 \%$. Assim, por exemplo, o Ministério da Educação coloca a taxa de abandono da coorte de 2009-2010 para estudos de graduação em 32\%, observando, no entanto, que isso é maior em determinados ramos do conhecimento, como Artes e Humanidades ou Engenharia e Arquitetura. E, embora seja verdade que essa taxa parece vir a diminuir, porque a taxa de abandono precoce (abandono no $1^{\circ}$ ano) da coorte 2011/2012 é de 22,5\% (MECD, 2016) e no ano de 2015/2016 é de 21,8\% (Fundación CYD, 2018), os resultados ainda estão longe do horizonte de 10\% estabelecido pela Estratégia Europa 2020 (Comissão Europeia, 2010).

No Brasil, a situação parece ser ainda mais preocupante. Segundo os dados do Censo da Educação Superior de 2015, realizado pelo Ministério da Educação, a taxa de abandono acumulada dos estudantes que ingressaram no ensino superior em 2010 foi, em média, de 49\%, sendo de 53\% nas particulares (onde o ensino superior é pago pelo estudante ou sua família) e $43 \%$ das públicas (municipais, estaduais ou federais, no qual o ensino é gratuito). Além disso, o mesmo levantamento mostrou que $30 \%$ da evasão ocorre até o segundo ano, havendo um decréscimo ao longo dos demais. É importante ressaltar também que, em 2015, a quantidade de matrículas novas no sistema de ensino superior brasileiro foi de 8.033.574 estudantes, sendo que $75 \%$ dos ingressantes estão em universidades particulares.

$\mathrm{Na}$ análise do abandono do ES importa atender aos diversos conceitos ou tipos de abandono e à complexidade ou multidimensionalidade dos fatores inerentes (Casanova Cervero, Nuñez, Bernardo, \& Almeida, 2018). O estudo das trajetórias dos estudantes, permitiu clarificar os diferentes tipos de abandono: estudantes em (a) stoptout - pausa por um período de tempo, até um ano, em que o estudante tem a intenção clara de retomar os estudos (Stratton, O'Toole, \& Wetzel, 2008); (b) optout mudança de curso ou de instituição em grande medida devido a questões vocacionais e ao fato de não ter ingressado no curso desejado, aproveitando as possibilidades de mobilidade dentro do sistema de ES (Montmarquette, Mahseredjian, \& Houle, 2001); e (c) dropout - abandono "definitivo" do ES. Essa será a situação mais penalizante para o estudante, uma vez que na maioria das vezes não está relacionada com uma decisão vocacional, mas sim uma impossibilidade de concretizar o seu projeto inicial de formação.

A complexidade do fenômeno está igualmente presente na diversidade e interação das causas associadas, emergindo vários modelos teóricos para sua explicação. O modelo ecológico/interacionista de Vicent Tinto (1975), seguramente, o mais referenciado internacionalmente, postula que o abandono ocorre quando os estudantes não se conseguem integrar social (interação com os seus pares na instituição) e academicamente (problemas de desempenho acadêmico e de desenvolvimento de carreira). A ênfase interacionista desse modelo entende o abandono como resultado de interações dinâmicas e recíprocas entre características pessoais do estudante, características formais e informais das IES, e características da comunidade em que o estudante está inserido (Casanova et al., 2018; Duarte, Ramos-Pires, \& Gonçalves, 2014; Meyer \& Marx, 2014).

Numa breve síntese da investigação disponível, o abandono do ES tende a ser mais frequente por parte dos estudantes no decurso do $1^{\circ}$ ano, estando nessa altura associado a variáveis acadêmicas, econômicas, sociais e institucionais, nomeadamente (Bernardo et al., 2017; Casanova, 2018; Esteban, Bernardo, Tuero, Cervero, \& Casanova, 2017). Essa multiplicidade de variáveis aparece na investigação associada aos motivos 
com que os estudantes do $1^{\circ}$ ano justificam a sua intenção e consumação do abandono. Em particular, a decisão de abandonar depende da forma e grau com que os estudantes superam os novos desafios colocados pelo ensino superior (Ainscough, Stewart, Colthorpe, \& Zimbardi, 2017; Allan, McKenna, \& Dominey, 2014; Martínez-López et al., 2014) e do nível de investimento que colocam na realização das tarefas acadêmicas (Crissman \& Upcraft, 2004; Hu \& Kuh, 2002; Santiago, Tremblay, Basri, \& Arnal, 2008). Por exemplo, os níveis de autonomia dos estudantes face às novas responsabilidades e os níveis de resiliência face às dificuldades encontradas na sua adaptação, bem como os sentimentos de mal-estar ou de otimismo daí decorrentes, são fatores apontados como relevantes no insucesso acadêmico e abandono (Allan et al., 2014; Beauvais, Stewart, DeNisco, \& John, 2014; Holdsworth, Turner, \& Scott-Young, 2017; Li, Cao, Cao, \& Liu, 2015; Tinto, 2017; Wilkins, Butt, Kratochvil, \& Balakrishnan, 2016). Essas dificuldades podem estar mais presentes, e assim justificar taxas mais elevadas de abandono, junto dos estudantes provenientes de famílias sem tradição de frequência do ensino superior, de zonas rurais ou pertencentes a grupos socioeconômicos desfavorecidos (Bui, 2002; Collier \& Morgan, 2008; Lohfink \& Paulsen, 2005; Xu, 2007).

Essa pluralidade de motivos está patente em instrumentos usados internacionalmente na investigação sobre os fenômenos do abandono (Ambiel, Santos, \& Dalbosco, 2016), bem como em resultados de estudos ex post facto sobre os motivos apontados pelos estudantes para a sua decisão de abandonar a formação (Bernardo et al., 2017; Esteban et al., 2017). A investigação sugere que tomando um modelo multidimensional e precoce de identificação de estudante em risco de abandono possibilita a prevenção do fenômeno (Ortiz-Lozano, Rua-Vieites, Bilbao-Calabuig, \& Casadesús-Fa, 2018). Sendo o $1^{\circ}$ ano crítico em termos da adaptação e permanência dos estudantes no ES, em boa medida por causa da diversidade de fatores estressantes vivenciados pelos estudantes na sua transição para o ES (GarcíaRos, Pérez-González, Pérez-Blasco, \& Natividad, 2012; Martínez-López et al., 2014), a identificação do risco de abandono deve ocorrer já nos primeiros tempos na universidade (Wilson et al., 2016).

Face à relevância do problema e às possibilidades de se intervir na sua prevenção mediante conhecimento atempado dos fatores de risco associados à decisão de abandono por parte dos estudantes, uma equipe de investigadores da Universidade do Minho (Portugal), da Universidade de Oviedo (Espanha) e da Universidade de São Francisco (Brasil) envolveram-se num projeto de investigação tendo em vista a construção e validação de um questionário de avaliação transcultural dos motivos de abandono. Buscando maior relevância e validade ecológica do questionário, a construção dos itens tomou uma perspetiva multidimensional dos motivos, baseando-nos na literatura internacional, em estudos realizados em cada país e na auscultação de grupos de estudantes nas três universidades indicadas sobre a intenção e a decisão do abandono (focus group, reflexão falada, questões abertas). Particular atenção nessa construção foi dada à estrutura dimensional e aos itens formulados na Escala de Motivos para Evasão do Ensino Superior (Ambiel, 2015), uma vez que esse instrumento se mostrou como pioneiro na avaliação dos motivos, de forma específica. Além disso, justifica-se a opção de desenvolver novo instrumento pelo fato de que no estudo de Ambiel (2015) os itens e dimensões do instrumento foram desenvolvidos considerando exclusivamente estudos brasileiros sobre o assunto que tiveram anteriormente o objetivo de avaliar os motivos para evasão.

Dessa forma, ainda que tenha servido como base de literatura para o atual trabalho, considerou-se que um novo instrumento, abrangendo desde o início as peculiaridades e semelhanças entre os países em questão, seria uma melhor decisão. Neste estudo, descrevem-se os procedimentos conducentes à primeira versão do questionário e os resultados obtidos com a sua aplicação junto a amostras de estudantes do $1^{\circ}$ ano nos três países. Basicamente, examinou-se o funcionamento dos itens e a estrutura fatorial do questionário em cada um dos três países, tendo em vista a identificação de modificações a introduzir numa nova versão mais definitiva do questionário.

\section{Método}

\section{Participantes}

Participaram no estudo 1.162 estudantes do $1^{\circ}$ ano do Ensino Superior, sendo 333 estudantes a frequentar o ES em Portugal (28,7\%), 287 estudantes a frequentar o ES em Espanha (24,7\%) e 542 estudantes a frequentarem o ES no Brasil (46,6\%). Em Portugal e Espanha, os estudantes são provenientes de instituições públicas e no Brasil de uma instituição privada. Considerando o total de participantes, 347 são do sexo masculino (30,3\%) e 797 estudantes são do sexo feminino $(69,7 \%)$, com idades compreendidas entre os $16 \mathrm{e}$ $64(M=21,6, D P=6,34)$. A maioria dos estudantes refere frequentar o curso de primeira opção de escolha $(75,4 \%)$, assim como a universidade de primeira opção de escolha $(76,0 \%)$.

Referente aos estudantes de Portugal, $56,9 \%$ são do sexo feminino e $43,1 \%$ são do sexo masculino, sendo a média de idades de 20,33 anos $(D P=5,96)$. Os estudantes frequentavam os cursos de Economia, Educação, Educação Básica, História, Relações Internacionais, Engenharia Informática, sendo que $71,0 \%$ frequentavam o curso de primeira opção de escolha e $68,3 \%$ referem frequentar a universidade de primeira opção de escolha. Destes estudantes, 34,3\% referem já ter pensado em 
abandonar o curso que frequentam e 14,8\% referem já ter pensado em abandonar o Ensino Superior.

Relativamente aos estudantes de Espanha, 83,3\% são do sexo feminino e $16,71 \%$ são do sexo masculino, sendo a média de idades de 20,04 anos $(D P=4,84)$. Os estudantes frequentavam os cursos de Enfermagem, Educação Infantil e Primária, Psicologia, Logopedia, sendo que $75,8 \%$ frequentavam o curso de primeira opção de escolha e $94,7 \%$ referem frequentar a universidade de primeira opção de escolha. Destes estudantes, 28,0\% referem já ter pensado em abandonar o curso que frequentam e 14,3\% referem já ter pensado em abandonar o Ensino Superior.

No caso dos estudantes do Brasil, 70,3\% são do sexo feminino e $29,7 \%$ são do sexo masculino, sendo a média de idades de 23,14 anos $(D P=6,88)$. Os estudantes frequentavam os cursos Psicologia, Direito, Arquitetura, Administração de Empresas, Ciências Contábeis e Medicina Veterinária, sendo que $77,9 \%$ frequentavam o curso de primeira opção de escolha e 70,5\% referem frequentar a universidade de primeira opção de escolha. Destes estudantes, $35,8 \%$ referem já ter pensado em abandonar o curso que frequentam.

\section{Instrumentos}

Questionário de Motivos de Abandono do Ensino Superior. O Questionário de Motivos de Abandono do Ensino Superior, tomando a literatura na área e respostas a questões abertas junto de estudantes dos três países envolvidos no projeto, integra 55 itens reportados a situações de dificuldade, de desmotivação ou de mal-estar experienciadas pelos estudantes e que, eventualmente, os podem levar a pensar no abandono do curso ou dos estudos de nível superior. Estes itens repartiam-se por grandes áreas de motivos de abandono: (a) Sociais (e.g., Não me identifico com os colegas do meu curso ou instituição), (b) Acadêmicos (e.g., Tenho dificuldade em entender certas matérias das disciplinas); (c) Professores (e.g., Não existe feedback dos professores sobre a aprendizagem dos alunos); (d) Saúde e bem-estar (e.g., Não faço tanto exercício como desejaria); (e) Institucionais (e.g., Os serviços da minha instituição funcionam de forma deficitária); e (f) Financeiros (e.g., Preciso de um emprego a tempo parcial ou a tempo inteiro para suportar os mês gastos). A resposta a cada situação ou item foi dada numa escala Likert de 5 pontos, consoante o grau de importância atribuído, variando entre 1 - Nada importante e 5 - Muito importante.

\section{Procedimentos}

Os procedimentos de recolha de dados foram uniformizados nos três países que participaram no estudo e levados a cabo por profissionais certificados e experientes na área da Psicologia. A recolha de dados foi realizada após um semestre de frequência do ES, e o critério de seleção dos estudantes foi serem estudantes do $1^{\circ}$ ano do ES, tendo sido assegurados os procedimentos éticos exigidos em cada um dos países, sendo que no Brasil, o projeto foi aprovado por um comitê de ética. Foram asseguradas as autorizações para o recolhimento de dados junto dos estudantes, sendo que as aplicações ocorreram em sala de aula. Aos estudantes foram apresentados os objetivos de investigação, assegurando o seu direito à não participação e a confidencialidade dos dados recolhidos. Os estudantes deram o seu consentimento livre e esclarecido para a participação no estudo, decorrendo a aplicação coletiva em contexto de sala de aula.

\section{Procedimento de Análise de dados}

As análises estatísticas dos resultados nos itens foram realizadas por recurso ao IBM/SPSS, versão 24.0. As análises estatísticas centraram-se no estudo da dimensionalidade e consistência interna das respostas aos itens. Realizou-se a análise fatorial exploratória (AFE), sendo inicialmente verificado se os dados eram passíveis de fatoração. Para tanto, recorreu-se aos métodos Kaiser-Meyer-Olkin (KMO) e ao Teste de Esfericidade de Barlett. Para a análise da confiabilidade da estrutura fatorial recorreu-se ao alfa de Cronbach. A respeito de dados omissos (missing), esses casos foram muito residuais, nunca chegando a $5 \%$ dos respondentes e apenas em alguns itens e, portanto, os protocolos foram mantidos na base e os valores mantiveram-se em branco.

\section{Resultados}

Realizou-se uma análise fatorial exploratória com os 55 itens, para cada país, por meio do método de componentes principais, com rotação varimax. Analisando a estrutura fatorial do instrumento em cada país, e com o objetivo de alcançar uma estrutura comum aos três países, selecionaram-se os itens comuns aos três países com melhor saturação em cada fator (acima de ,40). Dessa forma, excluímos 22 itens e realizamos uma nova análise fatorial com 32 itens, com o método dos componentes principais, forçando a 6 fatores (número equivalente à formulação teórica dos conteúdos a abranger na escala). Na Tabela 2 descrevemos os agrupamentos dos itens em cada um dos três países e níveis de saturação obtidos. Previamente, apreciando a factorização dos itens em cada país, obtiveram-se os seguintes índices: Portugal $(\mathrm{KMO}=, 921$; teste de esfericidade de Bartlett $=5904,353, g l=496$, $p=, 000$; variância explicada pelos primeiros seis fatores $=63,09 \%)$, Espanha $(\mathrm{KMO}=, 916$; teste de esfericidade de Bartlett $=5124,068, g l=496, p=, 000$; variância explicada pelos primeiros seis fatores $=61,90 \%$ ), e Brasil $(\mathrm{KMO}=, 934$; teste de esfericidade de Bartlett $=8086,429$, $g l=496, p=, 000$; variância explicada pelos primeiros seis fatores $=58,79 \%$ ). 
Tabela 1

Distribuição dos Itens pelos Fatores em Portugal (PT), Espanha (ES) e Brasil (BR)

\begin{tabular}{|c|c|c|c|c|c|c|c|c|c|}
\hline \multirow{2}{*}{ Itens } & \multicolumn{3}{|c|}{ Financeiro } & \multicolumn{3}{|c|}{ Institucional } & \multicolumn{3}{|c|}{ Académico } \\
\hline & PT & ES & $\mathrm{BR}$ & $\mathrm{PT}$ & ES & $\mathrm{BR}$ & $\mathrm{PT}$ & ES & $\mathrm{BR}$ \\
\hline 45. Tenho dificuldade em pagar as taxas para frequentar a universidade. & 0,83 & 0,84 & 0,55 & & & & & & \\
\hline 39. Tenho dificuldade em custear as minhas despesas da universidade. & 0,81 & 0,82 & 0,69 & & & & & & \\
\hline $\begin{array}{l}\text { 34. Não tenho valor de bolsa ou apoios suficientes para fazer } \\
\text { frente aos gastos na universidade. }\end{array}$ & 0,79 & 0,82 & 0,53 & & & & & & \\
\hline $\begin{array}{l}\text { 15. Preciso de um emprego a tempo parcial ou tempo inteiro para } \\
\text { suportar os meus gastos. }\end{array}$ & 0,78 & 0,72 & 0,70 & & & & & & \\
\hline 14. Tenho que cuidar da minha família. & 0,67 & 0,69 & 0,59 & & & & & & \\
\hline 2. Os serviços da minha instituição funcionam de forma deficitária. & & & & 0,63 & 0,45 & 0,71 & & & \\
\hline 5. As salas de aula não estão cuidadas. & & & & 0,77 & 0,65 & 0,75 & & & \\
\hline 6. A reputação da minha instituição no exterior é fraca. & & & & 0,71 & 0,65 & 0,65 & & & \\
\hline 10. Faltam serviços de apoio a estudantes em dificuldade. & & & & 0,51 & 0,45 & 0,52 & & & \\
\hline 11. Os laboratórios, bibliotecas ou equipamentos não estão atualizados. & & & & 0,75 & 0,70 & 0,66 & & & \\
\hline 19. Tenho dificuldade em entender certas matérias das disciplinas. & & & & & & & 0,64 & 0,64 & 0,69 \\
\hline 22. Estou a ter baixo rendimento académico. & & & & & & & 0,74 & 0,74 & 0,55 \\
\hline 25. Custa-me realizar os trabalhos de grupo. & & & & & & & 0,51 & 0,51 & 0,58 \\
\hline 26. Vou deixar disciplinas em atraso. & & & & & & & 0,67 & 0,67 & 0,71 \\
\hline 27. Não tenho tempo suficiente para estudar como desejaria. & & & & & & & 0,60 & 0,60 & 0,63 \\
\hline 35. O método de ensino dos professores não me agrada. & & & & & & & & & \\
\hline $\begin{array}{l}\text { 36. Os conteúdos que os professores ensinam não estão atualizados } \\
\text { ou não têm profundidade. }\end{array}$ & & & & & & & & & \\
\hline $\begin{array}{l}\text { 37. Os professores não atendem às observações que fazem os alunos } \\
\text { sobre as suas aulas. }\end{array}$ & & & & & & & & & \\
\hline 46. Os professores não se interessam pela situação pessoal dos alunos. & & & & & & & & & \\
\hline 51. Não existe uma relação próxima entre professores e alunos. & & & & & & & & & \\
\hline 55. A organização e funcionamento do meu curso não me agradam. & & & & & & & & & \\
\hline 17. Não tenho amigos nesta universidade & & & & & & & & & \\
\hline 29. Não me identifico com os colegas do meu curso ou instituição. & & & & & & & & & \\
\hline 32. Não me agrada a região ou a cidade onde está a minha universidade. & & & & & & & & & \\
\hline 44. Não consegui integrar-me socialmente na universidade. & & & & & & & & & \\
\hline $\begin{array}{l}\text { 47. Não me identifico com as pessoas da cidade onde está a minha } \\
\text { universidade. }\end{array}$ & & & & & & & & & \\
\hline 49. Desagrada-me o alojamento onde resido durante as aulas. & & & & & & & & & $0,48 b$ \\
\hline 16. Não faço tanto exercício físico como desejaria. & & & & & & & & & \\
\hline 23. Meus hábitos alimentares não são adequados. & & & & & & & & & \\
\hline 31. Não participo nas festas ou atividades dos estudantes. & & & & & & & & & \\
\hline 42. Não me agrada a alimentação que faço em tempo de aulas. & & & & & & & & & \\
\hline 50. Não durmo o tempo necessário para render no estudo. & & & & & & & & & \\
\hline
\end{tabular}

\begin{tabular}{|c|c|c|c|c|c|c|c|}
\hline \multirow{2}{*}{ Itens } & \multicolumn{3}{|c|}{ Professores } & \multicolumn{3}{|c|}{ Social } & Bem-estar \\
\hline & PT & ES & $\mathrm{BR}$ & PT & ES & $\mathrm{BR}$ & $\begin{array}{lll}\text { PT } & \text { ES } & \text { BR }\end{array}$ \\
\hline
\end{tabular}

45. Tenho dificuldade em pagar as taxas para frequentar a universidade.

39. Tenho dificuldade em custear as minhas despesas da universidade.

34. Não tenho valor de bolsa ou apoios suficientes para fazer frente aos gastos na universidade. 
Tabela 1 (continuação) Distribuição dos Itens pelos Fatores em Portugal (PT), Espanha (ES) e Brasil (BR)

\begin{tabular}{|c|c|c|c|c|c|c|c|c|c|}
\hline \multirow{2}{*}{ Itens } & \multicolumn{3}{|c|}{ Professores } & \multicolumn{3}{|c|}{ Social } & \multicolumn{3}{|c|}{ Bem-estar } \\
\hline & $\mathrm{PT}$ & ES & $\mathrm{BR}$ & $\mathrm{PT}$ & ES & $\mathrm{BR}$ & $\mathrm{PT}$ & ES & $\mathrm{BR}$ \\
\hline $\begin{array}{l}\text { 15. Preciso de um emprego a tempo parcial ou tempo inteiro para } \\
\text { suportar os meus gastos. }\end{array}$ & & & & & & & & & \\
\hline 14. Tenho que cuidar da minha família. & & & & & & & & & \\
\hline 2. Os serviços da minha instituição funcionam de forma deficitária. & & & & & & & & & \\
\hline 5. As salas de aula não estão cuidadas. & & & & & & & & & \\
\hline 6. A reputação da minha instituição no exterior é fraca. & & & & & & & & & \\
\hline 10. Faltam serviços de apoio a estudantes em dificuldade. & & & & & & & & & \\
\hline 11. Os laboratórios, bibliotecas ou equipamentos não estão atualizados. & & & & & & & & & \\
\hline 19. Tenho dificuldade em entender certas matérias das disciplinas. & & & & & & & & & \\
\hline 22. Estou a ter baixo rendimento académico. & & & & & & & & & \\
\hline 25. Custa-me realizar os trabalhos de grupo. & & & & & & & & & \\
\hline 26. Vou deixar disciplinas em atraso. & & & & & & & & & \\
\hline 27. Não tenho tempo suficiente para estudar como desejaria. & & & & & & & & & \\
\hline 35. O método de ensino dos professores não me agrada. & 0,68 & 0,78 & 0,51 & & & & & & \\
\hline $\begin{array}{l}\text { 36. Os conteúdos que os professores ensinam não estão atualizados } \\
\text { ou não têm profundidade. }\end{array}$ & 0,64 & 0,66 & 0,58 & & & & & & \\
\hline $\begin{array}{l}\text { 37. Os professores não atendem às observações que fazem os alunos } \\
\text { sobre as suas aulas. }\end{array}$ & 0,69 & 0,65 & 0,62 & & & & & & \\
\hline 46. Os professores não se interessam pela situação pessoal dos alunos. & 0,62 & 0,69 & 0,48 & & & & & & \\
\hline 51. Não existe uma relação próxima entre professores e alunos. & 0,61 & 0,70 & 0,62 & & & & & & \\
\hline 55. A organização e funcionamento do meu curso não me agradam. & 0,57 & 0,51 & 0,54 & & & & & & \\
\hline 17. Não tenho amigos nesta universidade & & & & 0,61 & 0,66 & 0,57 & & & \\
\hline 29. Não me identifico com os colegas do meu curso ou instituição. & & & & 0,61 & 0,52 & 0,62 & & & \\
\hline 32. Não me agrada a região ou a cidade onde está a minha universidade. & & & & 0,65 & 0,58 & 0,60 & & & \\
\hline 44. Não consegui integrar-me socialmente na universidade. & & & & 0,70 & 0,72 & 0,65 & & & \\
\hline $\begin{array}{l}\text { 47. Não me identifico com as pessoas da cidade onde está a minha } \\
\text { universidade. }\end{array}$ & & & & 0,67 & 0,69 & 0,68 & & & \\
\hline 49. Desagrada-me o alojamento onde resido durante as aulas. & & & & 0,57 & 0,57 & $0,47 b$ & & & \\
\hline 16. Não faço tanto exercício físico como desejaria. & & & & & & & 0,72 & 0,66 & 0,73 \\
\hline 23. Meus hábitos alimentares não são adequados. & & & & & & & 0,71 & 0,72 & 0,77 \\
\hline 31. Não participo nas festas ou atividades dos estudantes. & & & & & $0,56 a$ & $0,44 \mathrm{c}$ & 0,47 & $0,47 a$ & $0,43 c$ \\
\hline 42. Não me agrada a alimentação que faço em tempo de aulas. & & & & & & & 0,75 & 0,80 & 0,72 \\
\hline 50. Não durmo o tempo necessário para render no estudo. & & & & & & & 0,51 & 0,50 & 0,58 \\
\hline
\end{tabular}

Como pode-se ver na Tabela 1, os itens distribuem-se pelos fatores da mesma forma, à exceção dos itens 31 (Não participo nas festas ou atividades dos estudantes.) e 49 (Desagrada-me o alojamento onde resido durante as aulas.). O item 49 em Portugal e Espanha surge no fator Social. No entanto, no Brasil apresenta maior saturação no fator Acadêmico $(48)$, embora com valor próximo $(, 47)$ no fator Social. Relativamente ao item 31, na Espanha integra o fator social $(, 56)$, assim como no Brasil $(, 44)$, no entanto em Portugal integra o fator do Bem-estar, ocorrendo também no Brasil a sua saturação neste segundo fator $(, 44)$. Em futuros estudos, torna-se necessário definir melhor o conteúdo destes dois itens de forma a que assumam um significado mais claramente vinculado ao fator Social.

$\mathrm{Na}$ Tabela 2 apresentamos as médias e desvio padrão dos resultados nas seis dimensões bem como os índices de consistência interna dos itens agrupados em 
cada dimensão. Em relação às médias obtidas nos três países (sendo importante ponderar o número de itens em cada dimensão para comparação entre si), observam-se valores mais baixos na amostra brasileira, podendo sugerir que tais motivos estão atenuados quando os estudantes frequentam uma instituição provada de ensino superior. Entre Portugal e Espanha a maior diferença ocorre nos motivos financeiros, havendo uma média mais elevada em Espanha, podendo refletir a existência de menores apoios sociais (bolsas de estudo). Relativamente aos dois itens que apresentaram saturações em mais que um fator (item 31 e item 49), a opção passou pela sua inclusão apenas na dimensão em que era teoricamente esperado.

Tabela 2

Consistência Interna dos Itens (Alfa de Cronbach) por País

\begin{tabular}{|c|c|c|c|c|c|c|c|c|c|c|}
\hline \multirow{2}{*}{ Fatores } & \multirow{2}{*}{$\mathrm{N}^{\circ}$ itens } & \multicolumn{3}{|c|}{ Portugal } & \multicolumn{3}{|c|}{ Espanha } & \multicolumn{3}{|c|}{ Brasil } \\
\hline & & $\mathrm{M}$ & $\mathrm{DP}$ & $\alpha$ & $\mathrm{M}$ & $\mathrm{DP}$ & $\alpha$ & $\mathrm{M}$ & $\mathrm{DP}$ & $\alpha$ \\
\hline Social & 6 & 15,98 & 5,59 & 0,86 & 15,97 & 5,33 & 0,85 & 12,09 & 5,28 & 0,86 \\
\hline Saúde e Bem-estar & 5 & 12,20 & 4,31 & 0,78 & 12,04 & 4,09 & 0,75 & 11,64 & 4,65 & 0,78 \\
\hline Institucional & 5 & 13,76 & 4,63 & 0,83 & 13,61 & 3,78 & 0,74 & 13,19 & 5,16 & 0,83 \\
\hline Financeiros & 5 & 16,76 & 5,79 & 0,90 & 18,69 & 5,45 & 0,91 & 15,36 & 5,60 & 0,90 \\
\hline Académicos & 5 & 15,68 & 4,37 & 0,82 & 15,72 & 4,16 & 0,81 & 13,40 & 4,70 & 0,82 \\
\hline Professores & 6 & 17,81 & 5,27 & 0,85 & 18,57 & 5,06 & 0,86 & a) & a) & a) \\
\hline
\end{tabular}

Nota. a) não calculada em virtude deste fator não ter sido devidamente identificado na amostra do Brasil

Relativamente à consistência interna dos itens, os valores de alfa de Cronbach obtidos são superiores a,70 tomando o conjunto de itens por dimensão da escala em cada país. Lembrando que a dimensão reportada aos comportamentos, atitudes ou falta de apoio dos professores deverá ser de novo aplicada no Brasil, nomeadamente recorrendo a instituições públicas de ensino superiores, antecipando relações professor-aluno diferenciadas, tomando instituições públicas e privadas de Ensino Superior.

\section{Discussão e Considerações Finais}

Os resultados obtidos quanto à validade e precisão dos resultados nos 32 itens retidos permitem identificar seis fatores satisfatoriamente representados nos três países: (a) Social (motivos relacionados com a interação e integração social), (b) Acadêmico (motivos relacionados com o desempenho e rendimento académico); (c) Professores (motivos relacionados com os métodos e relacionamento com os professores); Saúde e bem-estar (motivos relacionados com a alimentação, sono e bem-estar); Institucional (motivos relacionados com as características das instituições), e Financeiro (motivos relacionados com dificuldades financeiras para pagar despesas inerentes). A distribuição dos itens pelas seis dimensões, estando estas diferenciadas entre si, apontam para a diversidade de motivos que podem estar na origem da intenção de abandono do ensino superior pelos estudantes. Esta diversidade atende à multimensionalidade dos fatores explicativos do abandono, em particular quando se trabalha com estudantes do $1 .^{\circ}$ ano em que as taxas de abandono do ES são mais elevadas (Almeida et al.,
2008; Bernardo et al., 2017; Casanova, 2018; Esteban et al., 2017; Matta, Lebrão, \& Heleno, 2017; Tinto, 2012). Reportando-nos, ainda, à dimensionalidade do questionário, a procura de uma amostra mais heterogénea de estudantes em termos de instituições de ensino superior no Brasil ajudará a clarificar as dificuldades encontradas na validação da dimensão relativa às dificuldades no relacionamento dos estudantes com os seus professores, como potencial motivo de abandono. Os valores de alfa de Cronbach são também adequados, atingindo índices superiores a 0,70, assumindo-se assim bons níveis de confiabilidade por meio da consistência interna dos itens nas várias dimensões da escala para os três países, excluindo a dimensão "Professores" em relação ao Brasil pelas razões já apontadas. Esta situação particular da amostra brasileira pode ter diversas origens que importam clarificar, inclusive orientando a composição das amostras nos próximos estudos. Para além de uma eventual variável cultural, desde logo merece referência o facto da amostra ter sido coletada em instituição privada. Esta condição pode diferenciar os dados face às amostras nos outros dois países em que se recorreu a instituições públicas.

Em relação aos resultados aqui encontrados em comparação com achados prévios da literatura, vale um comentário à luz do que já foi relatado por Ambiel (2015), quanto aos motivos em amostras brasileiras. Nota-se que há alguns pontos de convergência entre os achados, por exemplo, o fato de que ambos os instrumentos terem dimensões específicas para a avaliação de motivos relativos à integração e intereção social, motivos institucionais, motivos relativos à dificuldades financeiras e quanto a aspectos acadêmicos. Por outro lado, no estudo transcultural, foi identificada uma 
dimensão relacionada a Saúde e Bem-estar, que não havia sido previamente identificada pelo instrumento brasileiro. Da mesma forma, a dimensão que neste estudo foi denominada Professores também não encontrou consonância no Brasil, nem neste mesmo estudo transcultural, tampouco no estudo anterior de Ambiel (2015). Ainda neste sentido, Ambiel (2015) identificou duas dimensões que não foram abordados no presente estudo, referentes aos motivos relativos à carreira e os vocacionais.

Pensando nas limitações do presente estudo, uma nova amostra nos três países é relevante para se validar de novo a escala, recorrendo à análise fatorial confirmatória dos seus itens. Complementarmente, é também necessário que se faça o estudo da invariância dos parâmetros do instrumento em relação às amostras dos diferentes países, até para melhor se entenderem as oscilações verificadas nas médias nas seis dimensões ao nível das amostras dos três países. Essa amostra deve ser suficientemente heterogénea em termos de instituições e de cursos para dar maior segurança e representatividade aos dados obtidos, continuando a privilegiar os estudantes que frequentam o $1^{\circ}$ ano. Nessa nova aplicação é fundamental incluir algumas outras variáveis psicossociais e de rendimento académico para servirem o objetivo da validade de critério (validade externa).

\section{Referências}

Ainscough, L., Stewart, E., Colthorpe, K., \& Zimbardi, K. (2017). Learning hindrances and self-regulated learning strategies reported by undergraduate students: Identifying characteristics of resilient students. Studies in Higher Education, 8(21), 1-16. doi:10.1080/0307507 9.2017.1315085

Allan, J. F., McKenna, J., \& Dominey, S. (2014). Degrees of resilience: Profiling psychological resilience and prospective academic achievement in university inductees. British Journal of Guidance \& Counselling, 42(1), 9-25. doi:10.1080/03069885.2013.793784

Almeida, L. S., Vasconcelos, R., \& Mendes, T. (2008). O abandono dos estudantes no Ensino Superior: Um estudo na Universidade do Minho. Revista Galego-Portuguesa de Psicoloxía e Educación, 16(1,2), 109-117. Recuperado de http://hdl.handle.net/1822/26571

Ambiel, R. A. M. (2015). Development of the reasons for Higher Education Dropout Scale. Revista Avaliação Psicológica, 14(1), 41-52. doi:10.15689/ap.2015.1401.05

Ambiel, R. A. M., Santos, A. A. A., \& Dalbosco, S. N. P. (2016). Motivos para evasão, vivências acadêmicas e adaptabilidade de carreira em universitários. Psico, 47(4), 288. doi:10.15448/1980-8623.2016.4.23872

Beauvais, A. M., Stewart, J. G., DeNisco, S., \& John, E. (2014). Factors related to academic success among nursing students: A descriptive correlational research study. Nurse Education Today, 34(6), 918-923. doi:10.1016/j.nedt.2013.12.005

Bernardo, G. A. B., Cervero, A., Esteban, M., Tuero, E., Casanova, J. R., \& Almeida, L. S. (2017). Freshmen program withdrawal: Types and recommendations. Frontiers in Psychology, 8(September). doi:10.3389/fpsyg.2017.01544

Bui, K. V. T. (2002). First-generation college students at a four-year university: Background characteristics, reasons for pursuing higher education, and first-year experiences. College Student Journal, 36(1), 3-12.

Casanova, J. R. (2018). Abandono no ensino superior: Modelos teóricos, evidências empíricas e medidas de intervenção. Educação: Teoria e Prática, 28(57), 5-22. doi:10.18675/1981-8106.vol28.n57.p05-22

Casanova, J. R., Cervero, A., Nuñez, J. C., Bernardo, A. B., \& Almeida, L. S. (2018). Abandono no Ensino Superior: Impacto da autoeficácia na intenção de abandono. Revista Brasileira de Orientação Profissional, 19(1), 41-49. doi:1026707/1984-7270/2019v19n1p41

Collier, P. J., \& Morgan, D. L. (2008). "Is that paper really due today?" Differences in first-generation and traditional college students' understandings of faculty expectations. Higher Education, 55(4), 425-446. doi: 10.1007/s10734-007-9065-5

Comissão Europeia. (2010). Comunicação da Comissão. Europa 2020: Estratégia para um crescimento inteligente, sustentável e inclusivo. Bruxelas: Comissão Europeia. Recuperado de https://eur-lex.europa.eu/legal-content/PT/TXT/?uri=celex:52010DC2020

Crissman, J. L., \& Upcraft, M. L. (2004). The keys to first-year student persistence. Em M. L. Upcraft, J. N. Gardner, \& B. O. Barefoot (Eds.), Challenging and supporting the first year student (pp. 27-46). San Francisco: Jossey-Bass.

Duarte, R., Ramos-Pires, A., \& Gonçalves, H. (2014). Identifying at-risk students in higher education. Total Quality Management $\mathcal{E}$ Business Excellence, 25(7-8), 944-952. doi:10.1080/14783363.2014.906110

Engrácia, P., \& Baptista, J. (2018). Percursos no Ensino Superior: Situação após quatro anos dos alunos inscritos em licenciaturas de três anos. DGEEC: Lisboa.

Esteban, M., Bernardo, G. A. B., Tuero, E., Cervero, A., \& Casanova, J. R. (2017). Variables influyentes en progreso académico y permanencia en la universidad. European Journal of Education and Psychology, 8(1), 37-52. doi:10.1016/j.ejeps.2017.07.003

European Commission. (2015). Dropout and completion in higher education in Europe. Recuperado de http://supporthere.org/sites/default/files/ dropout-completion-he_en.pdf

Eurostat. (2018). Eurostat regional yearbook. Luxemburgo. https://doi.org/10.2785/29084

Fundación CYD. (2018). Informe CYD 2017. Barcelona: Fundación Conocimiento y Desarrollo.

García-Ros, R., Pérez-González, F., Pérez-Blasco, J., \& Natividad, L. A. (2012). Evaluación del estrés académico en estudiantes de nueva incorporación a la universidade. Revista Latinoamericana de Psicología, 44(2), 143-154. Recuperado de http://www.redalyc.org/articulo. oa? id $=80524058011$

Holdsworth, S., Turner, M., \& Scott-Young, C. M. (2017). Not drowning, waving. Resilience and university: A student perspective. Studies in Higher Education, 43(11), 1837-1853. doi: 10.1080/03075079.2017.1284193

Hu, S., \& Kuh, G. D. (2002). Being (Dis) engaged in educationally purposeful activities: The influences of student and institutional characteristics. Research in Higher Education, 43(5), 555-575. doi: 10.1023/A:1020114231387 
Li, Y., Cao, F., Cao, D., \& Liu, J. (2015). Nursing students' post-traumatic growth, emotional intelligence and psychological resilience. Journal of Psychiatric and Mental Health Nursing, 22(5), 326-332. doi:10.1111/jpm.12192

Lohfink, M. M., \& Paulsen, M. B. (2005). Comparing the determinants of persistence for first-generation and continuing-generation students. Journal of College Student Development, 46(4), 409-428. doi: 10.1353/csd.2005.0040

Martínez-López, Z., Páramo-Fernández, M. F., Guisande-Couñago, M. A., Tinajero-Vacas, C., Almeida, L. S., \& Rodríguez-González, M. S. (2014). Apoyo social en universitarios españoles de primer año: propiedades psicométricas del Social Support Questionnaire-Short Form y el Social Provisions Scale. Revista Latinoamericana de Psicología, 46(2), 102-110. Recuperado de http://www.redalyc.org/articulo. oa?id $=80532608004$

Matta, C. M. B., Lebrão, S. M. G., \& Heleno, M. G. V. (2017). Adaptação, rendimento, evasão e vivências acadêmicas no ensino superior: revisão da literatura. Psicologia Escolar e Educacional, 21(3), 583-591. doi: 10.1590/2175-353920170213111118

MECD. (2016). Datos y cifras del del sistema universitario español. Curso 2015-2016. Madrid: Secretaría General Técnica.

Meyer, M., \& Marx, S. (2014). Engineering dropouts: A qualitative examination of why undergraduates leave engineering. Journal of Engineering Education, 103(4), 525-548. doi:10.1002/jee.20054

Montmarquette, C., Mahseredjian, S., \& Houle, R. (2001). The determinants of university dropouts: A bivariate probability model with sample selection. Economics of Education Review, 20(5), 475-484. doi: 10.1016/S0272-7757(00)00029-7

Ortiz-Lozano, J. M., Rua-Vieites, A., Bilbao-Calabuig, P., \& Casadesús-Fa, M. (2018). University student retention: Best time and data to identify undergraduate students at risk of dropout. Innovations in Education and Teaching International, 1-12. doi: 10.1080/14703297.2018.1502090

Santiago, P., Tremblay, K., Basri, E., \& Arnal, E. (2008). Tertiary education for the knowledge society. París: OCDE.

Stratton, L. S., O'Toole, D. M., \& Wetzel, J. N. (2008). A multinomial logit model of college stopout and dropout behavior. Economics of Education Review, 27(3), 319-331. doi: 10.1016/j.econedurev.2007.04.003

Tinto, V. (1975). Dropout from Higher Education: A Theoretical synthesis of recent research. Review of Educational Research, 45(1), 89-125. doi: $10.3102 / 00346543045001089$

Tinto, V. (2012). Completing college: Rethinking institutional action. Chicago, IL: The University of Chicago Press.

Tinto, V. (2017). Through the eyes of students. Journal of College Student Retention: Research, Theory E Practice, 19(3), 254-269. doi: $10.1177 / 1521025115621917$

Wilkins, S., Butt, M. M., Kratochvil, D., \& Balakrishnan, M. S. (2016). The effects of social identification and organizational identification on student commitment, achievement and satisfaction in higher education. Studies in Higher Education, 41(12), $2232-2252$

Wilson, K. L., Murphy, K. A., Pearson, A. G., Wallace, B. M., Reher, V. G. S., \& Buys, N. (2016). Understanding the early transition needs of diverse commencing university students in a health faculty: Informing effective intervention practices. Studies in Higher Education, 41(6), 1023-1040. doi: 10.1080/03075079.2014.966070

$\mathrm{Xu}, \mathrm{X}$. (2007). Cultural differences and socialization of rural undergraduates. Journal of China University of Mining E Technology (Social Science), $1,87-91$.

\section{Sobre os autores}

Leandro S. Almeida é psicólogo, doutor em Psicologia da Educação pela Universidade do Porto, professor Catedrático da Universidade do Minho, cordenador do Observatório dos Percursos Académicos dos Estudantes e membro do Centro de Investigação em Educação (CIEdUMinho, Portugal).

Joana R. Casanova é psicóloga, doutoranda em Ciências da Educação, especialidade de Psicologia da Educação com financiamento da Fundação para a Ciência e Tecnologia (SFRH/BD/117902/2016) e membro do Centro de Investigação em Educação (CIEd-UMinho, Portugal).

Ana B. Bernardo é doutora em Psicologia pela Universidade de Oviedo e professora na área de Psicologia Evolucionária e Educação no Departamento de Psicologia da Universidade de Oviedo.

António Cervero é graduado em Psicologia pela UNED, bacharel em Educação e Diploma em Educação pela Universidade de Oviedo e PDI (pessoal de ensino e pesquisa) da Universidade de Oviedo por meio do programa de bolsas de estudo pré-doutoral Severo Ochoa do Governo do Principado das Astúrias.

Acácia A. Angeli dos Santos é psicóloga, doutora em Psicologia Escolar e do Desenvolvimento Humano pelo Instituto de Psicologia da USP, professora titular do Programa de Pós-graduação Stricto Sensu em Psicologia da Universidade São Francisco, Campinas/Brasil e bolsista produtividade do CNPq.

Rodolfo A. M. Ambiel é psicólogo e doutor em Psicologia pela Universidade São Francisco. Atualmente é docente do Programa de Pósgraduação Stricto Sensu em Psicologia da Universidade São Francisco (área de concentração em Avaliação Psicológica) e do curso de graduação em Psicologia da mesma universidade, editor-chefe da Revista Psico-USF (Qualis A2), presidente da Associação Brasileira de Orientação Profissional (ABOP) - gestões 2015-2017 e 2017-2019 e bolsista produtividade do CNPq - Nível 2. 